\title{
MANEJO EN COLOMBIA DE EVIDENCIAS EXTRAÍDAS EN CIRUGÍA PARA MANTENER LA CADENA DE CUSTODIA
}

\section{CONSENSO DE EXPERTOS}

\author{
Naida Lizeth Beltrán Pérez.*
}

\section{Resumen}

Introducción: en Colombia el proceso de recuperación y manejo de evidencias se rige por las resoluciones 2.869 de 2003 y 6.394 de 2004 que establecen el manual de cadena de custodia. Su aplicación es de obligatorio cumplimiento para los funcionarios de policía judicial y quienes por su profesión o condición tengan contacto con evidencia incluyendo personal de salud. La Superintendencia Nacional de Salud y la Secretaría Distrital de Salud exigen a las instituciones prestadoras de servicios de salud (IPS), el levantamiento e implementación de los procesos relacionados con el mantenimiento de la cadena de custodia en la atención de pacientes que lo requieran, porque los descritos en el manual no cumplen con el manejo del material obtenido de los pacientes quirúrgicos. Objetivo: proponer normas sobre las evidencias médico legales en cirugía para preservar la cadena de custodia en Colombia. Métodos: consenso de expertos con técnica Delphi. Resultados: se establecieron 38 recomendaciones por tipo de evidencia (material balístico, elementos cortopunzantes, estupefacientes y pruebas biológicas) y de acuerdo con el momento quirúrgico (antes, durante y después).

Palabras clave: recuperación de evidencia, cadena de custodia, pacientes quirúrgicos.

\section{HANDLING OF SURGICALLY OBTAINED FORENSIC EVIDENCE TO MAINTAIN CHAIN OF CUSTODY IN COLOMBIA: AN EXPERT CONSENSUS}

\section{Abstract}

Introduction: In Colombia, the process of evidence collection and handling is regulated by Laws 2869 of 2003 and 6394 of 2004 which describe the chain of custody procedure manual. Police officers and those whose profession or condition, including healthcare professionals, includes handling forensic evidence, are required to observe these laws. The National Health Superintendent and Bogota's Department of Health require the Health Providing Institutions (IPS), to record and implement the procedures related with maintaining a chain of custody when treating such patients, for those described in the manual do not include how to handle material obtained in surgical procedures. Objective: to propose guidelines on handling surgically obtained forensic evidence in order to preserve chain of custody in Colombia. Methods: an expert consensus panel using the Delphi method. Results: 38 recommendations according to the type of evidence (bullets, knives, illegal drugs and biologic tests) and phase of the surgical procedure (before, during or after) were designed.

Key words: evidence recovery, chain of custody, surgical patients 


\section{Introducción}

Ante los altos índices de violencia en Colombia ${ }^{1}$ los profesionales de la salud en los servicios de urgencias y cirugía se enfrentan de manera permanente a la atención de pacientes víctimas de hechos violentos y por ende a la responsabilidad de aplicar el sistema de cadena de custodia, como lo determina el Código de Procedimiento Penal en su artículo $255^{2}$ debido a la relación o al contacto con elementos materia de prueba o evidencia física que puedan ser de utilidad en la investigación penal; de la misma manera la Superintendencia Nacional de Salud con la circular 049 del año $2008^{3}$, recuerda a todos los sujetos y entidades vigiladas que:"[...] Los particulares que por razón de su trabajo o por el cumplimento de las funciones propias de su cargo, en especial el personal de los servicios de salud que entren en contacto con elementos materiales probatorios y evidencia física, son responsables por su recolección, preservación y entrega a la autoridad correspondiente.[...]".

En Colombia se exige la implementación del sistema de cadena de custodia a partir de 2003 y desde entonces solo las entidades judiciales han participado en la documentación de los procedimientos de manejo de evidencia, los cuales fueron descritos y reglamentados mediante las resoluciones 2869 de $2003^{4}$ y 6394 de $2004^{5}$. A pesar de la información detallada en la normatividad, no se identifican acciones precisas para el manejo de la evidencia física en procedimientos quirúrgicos, lo cual es de vital importancia, debido a que en su recuperación se puede alterar la autenticidad por el contacto con los diversos dispositivos médicos utilizados durante la cirugía, la aplicación de las técnicas de asepsia y antisepsia, el contacto con soluciones y la posible contaminación cruzada.

En la literatura científica se ha identificado que países como Australia y Estados Unidos cuentan con experiencias en las cuales grupos de profesionales de la salud establecieron guías para el manejo de la evidencia forense en las instituciones de salud. Es el caso de Evans de la Association of periOperative Registered Nurses $(A O R N)$ de Estados Unidos que planteó la necesidad de proteger las puntas de las pinzas para la extracción de material balístico, debido a que este posee unas marcas únicas dejadas por el arma de la cual fue disparado y el instrumental quirúrgico cuenta con estrías que pueden generar nuevas que impiden realizar procedimientos de comparación. ${ }^{6}$

Aportes como éste pueden enriquecer la normatividad para que los profesionales de los equipos quirúrgicos sumen a sus prácticas los lineamientos que permitirán proteger la evidencia, favorecer los procesos de investigación judicial y cumplir con los actos mandatorios de la legislación colombiana. El presente estudio plantea una propuesta del manejo de elementos materia de prueba y/o evidencia física extraída en cirugía para mantener la cadena de custodia, formulada por un consenso de expertos. Es un tema que ha sido poco abordado por los profesionales de la salud en Colombia, siendo una necesidad imperante unificar conceptos y tomar experiencias exitosas de otras instituciones, para formular lineamientos del manejo de la evidencia física en el área quirúrgica.

\section{Materiales y métodos}

Se llevó a cabo un consenso formal de expertos con técnica Delphi ${ }^{7}$, sin la presencia física y permanecieron en el anonimato para evitar que las respuestas fueran influenciadas entre ellos y el mecanismo de contacto con el grupo de investigación fue por correo electrónico. Se seleccionaron cuatro expertos teniendo en cuenta el conocimiento en el área forense y la experiencia en el manejo de evidencias y del sistema de cadena de custodia.

Se diseñaron cinco instrumentos relacionados con el manejo de la evidencia forense que puede ser extraída en cirugía, cuatro se relacionan con el tipo de evidencia (material balístico, elementos cortopunzantes, muestras biológicas y estupefacientes). En cada uno los expertos diligenciaron el formato de acuerdo con las especificaciones solicitadas, como el procedimiento relacionado con la evidencia (recuperación, manipulación y embalaje) y el momento para llevar a cabo cada una de las recomendaciones descritas (antes, durante o después del procedimiento quirúrgico). Todos debían emitir la justificación de cada una de 
las recomendaciones. El quinto cuestionario incluyó las preguntas relacionadas con la información que debe registrarse y la entrega a las autoridades competentes.

Se realizó el primer envío de los cuestionarios con el instructivo de diligenciamiento respectivo. Al recibir las respuestas se realizó un análisis de tipo cualitativo y se construyó un documento con los resultados preliminares. Luego se enviaron los resultados de los cuestionarios en la segunda ronda, en la cual emitieron un juicio de valor frente a cada una de las recomendaciones. Se adoptaron como definitivas aquellas que obtuvieron el $75 \%$ de acuerdo entre los expertos.

\section{Resultados}

Se establecieron 38 recomendaciones para el manejo de la evidencia extraída en cirugía, de acuerdo con el tipo y el momento del procedimiento quirúrgico. Todos manifestaron que el punto de partida de la aplicación de las recomendaciones que se describen a continuación es el conocimiento del manual de cadena de custodia por parte del personal del quirófano y es necesaria la aplicación de las precauciones estándar de bioseguridad para la manipulación de la evidencia.

Teniendo en cuenta que había recomendaciones que se presentaron en todos los cuestionarios, se agruparon como consideraciones generales. Estas especificaciones buscan favorecer tanto la protección de la evidencia como el cumplimiento de los procedimientos administrativos requeridos por la normatividad vigente (Tabla1).

Se establecieron cinco recomendaciones para el manejo de material balístico como se muestra en la Tabla 2. En los casos en los cuales se atienden pacientes con heridas por arma de fuego, se recomienda que antes de la limpieza de las mismas se coloque un papel de filtro que permita la absorción de los fluidos y de las partículas; esto se realizará en la medida de lo posible, teniendo en cuenta que el procedimiento practicado en la institución de salud busca preservar la vida del paciente aún arriesgando la recuperación de la evidencia. Se formularon cuatro recomendaciones para elementos cortopunzantes, trece para estupefacientes y tres para material biológico como se muestra en las Tablas 3, 4 y 5.

\section{Tabla I. Consideraciones generales para el} manejo de la evidencia en cirugía

El personal de salud no debe considerar todos los elemen-

I tos materia de pruebas halladas como pertenencias u objetos personales.

Si las condiciones lo permiten, se debe hacer registro fotográfico o con video antes del procedimiento sobre el

2 lugar donde se encuentra el elemento probatorio y desde diferentes ángulos. Después del procedimiento se tomarán fotografías de las evidencias extraídas.

Las instituciones prestadoras de servicios de salud deben disponer con la debida anticipación de medios de embalaje como frascos, bolsas, cajas y otros, los cuales se seleccionan

3 de acuerdo con las características de la evidencia para facilitar la preservación y seguridad de lo recolectado, al igual que etiquetas $\circ$ rótulos y formatos que proporcionen la documentación de la cadena de custodia.

Para los elementos materia de prueba o evidencia de ori-

4 gen biológico nunca se reutilizan los recipientes para su recolección y embalaje.

5 Bajo ninguna circunstancia manipular las evidencias sin guantes.

6 Hacer el registro detallado de la ubicación en la historia clínica.

7 Es necesario no perder de vista el elemento materia de prueba.

Si como consecuencia de la manipulación a pesar de haber tenido el cuidado requerido se produjeron adherencias o se alteraron algunos rastros o huellas, debe dejarse constancia para su correspondiente descarte.

No reciba elementos materia de prueba o evidencia física

9 que no estén embalados, sellados o rotulados, pues el primer custodio debe realizar dicho procedimiento.

Es necesario establecer un protocolo de entrega de los elementos materia de prueba o evidencia física a las autoridades. Se recomienda que las IPS asignen a un funcionario en

I0 particular para que reporte el ingreso del paciente-víctima a la policía judicial o en su defecto a la primera autoridad del lugar, que se responsabilice de entregar las evidencias a las autoridades competentes.

La autoridad competente (policía judicial) debe traer or-

I den escrita solicitando la evidencia y se debe constatar el registro del traspaso del elemento materia de prueba a la policía judicial.

12 Existe un término de 48 horas para la entrega a la autoridad competente, siempre y cuando exista denuncia.

Mientras se haga la entrega a la autoridad competente, los

13 elementos materia de prueba deben estar en un lugar seguro (almacén transitorio de evidencias de la entidad de salud). 


\begin{tabular}{|c|c|c|}
\hline $\mathbf{N}^{\circ}$ & $\begin{array}{l}\text { Ibla 2. Rec } \\
\text { Momentos } \\
\text { quirúrgicos }\end{array}$ & $\begin{array}{l}\text { lendaciones para material } \\
\text { balístico } \\
\text { Recomendaciones }\end{array}$ \\
\hline I & Antes & $\begin{array}{l}\text { En caso tal de recibir un paciente con } \\
\text { prendas de vestir se tendrá cuidado de } \\
\text { no dañarlas y sí se encuentran húmedas } \\
\text { se extenderán en un sitio aislado para } \\
\text { dejar secar al medio ambiente. }\end{array}$ \\
\hline 2 & Antes & $\begin{array}{l}\text { Sobre las heridas producidas por arma } \\
\text { de fuego, antes de limpiarlas colocar } \\
\text { papel de filtro que permita la absorción } \\
\text { de todos los fluidos o partículas que se } \\
\text { encuentran alrededor por unos segun- } \\
\text { dos, se retira y se coloca en un sobre } \\
\text { de papel limpio para enviarlo como evi- } \\
\text { dencia. }\end{array}$ \\
\hline 3 & Durante & $\begin{array}{l}\text { Extraiga el proyectil evitando el uso del } \\
\text { instrumental y en caso de ser necesario } \\
\text { su uso proteja las puntas de las pinzas } \\
\text { (opcional con sonda nelaton), para evi- } \\
\text { tar generar marcas en el proyectil de- } \\
\text { bido a que cada arma al ser disparada } \\
\text { deja una huella única en el proyectil. }\end{array}$ \\
\hline 4 & Durante & $\begin{array}{l}\text { No lavar o limpiar los proyectiles, dejar } \\
\text { secar antes de embalarlos. }\end{array}$ \\
\hline 5 & Después & $\begin{array}{l}\text { Cada proyectil se debe embalar por } \\
\text { separado. En caso de hallar varios pro- } \\
\text { yectiles o fragmentos no los mezcle, } \\
\text { manéjelos y embálelos en forma inde- } \\
\text { pendiente. }\end{array}$ \\
\hline
\end{tabular}

\begin{tabular}{|c|c|l|}
\hline \multicolumn{2}{|c|}{ Tabla 3. Recomendaciones para elementos } \\
No \\
cortopunzantes \\
$\begin{array}{c}\text { Momentos } \\
\text { quirúrgicos }\end{array}$ & \multicolumn{1}{|c|}{ Recomendaciones } \\
\hline 2 & Antes & $\begin{array}{l}\text { Si el elemento cuenta con una porción en } \\
\text { la parte externa cúbralo antes de realizar } \\
\text { la asepsia prequirúrgica y déjelo de manera } \\
\text { permanente con un elemento estéril. }\end{array}$ \\
\hline 3 & Durante & $\begin{array}{l}\text { Procure no causar ninguna marca o defor- } \\
\text { midad. }\end{array}$ \\
\hline 4 & Durante & $\begin{array}{l}\text { No lavar ni limpiar el elemento materia de } \\
\text { prueba. }\end{array}$ \\
\hline Después & $\begin{array}{l}\text { Tome el arma por la punta o por los ex- } \\
\text { tremos del borde superior del mango. Para } \\
\text { el embalaje se recomienda utilizar láminas } \\
\text { de cartón que pueden asirse con cordeles } \\
\text { que lo aseguran al abrir dos orificios que } \\
\text { permitan su amarre o aseguramiento. Los } \\
\text { cordones se colocan en aquellos sitios del } \\
\text { elemento en los que se advierta que no } \\
\text { puedan dañar el micro-elemento. Una vez } \\
\text { asegurado se acomoda en una caja propor- } \\
\text { cional al tamaño. }\end{array}$ \\
\hline
\end{tabular}

\begin{tabular}{|c|c|c|}
\hline $\mathbf{N}^{\circ}$ & $\begin{array}{l}\text { Momentos } \\
\text { quirúrgicos }\end{array}$ & Recomendaciones \\
\hline 1 & Antes & $\begin{array}{l}\text { Establecer la localización anatómica exacta de los } \\
\text { estupefacientes. Tomar las imágenes diagnósticas } \\
\text { necesarias para determinar la ubicación y llegar a } \\
\text { ellos sin ocasionar ruptura. }\end{array}$ \\
\hline 2 & Antes & $\begin{array}{l}\text { Conserve las imágenes obtenidas como radiogra- } \\
\text { fías, ecografías, etc. }\end{array}$ \\
\hline 3 & Antes & $\begin{array}{l}\text { Se debe dar aviso a la unidad de fiscalía o de po- } \\
\text { licía judicial más cercana para que disponga de } \\
\text { personal técnico en sustancias controladas para } \\
\text { el recibo de los elementos. }\end{array}$ \\
\hline 4 & Durante & $\begin{array}{l}\text { Registre mediante fotografías en forma detallada } \\
\text { y progresiva la recuperación de los elementos, } \\
\text { dejando constancia de su número y estado en } \\
\text { ese momento. }\end{array}$ \\
\hline 5 & Durante & $\begin{array}{l}\text { Durante la extracción trate de no perforar el } \\
\text { embalaje de los elementos con el instrumental } \\
\text { que esté utilizando, pues deben ser examinados } \\
\text { por personal experto en las mismas condiciones } \\
\text { en que fueron hallados. Utilizar guantes o pinzas } \\
\text { de punta cubiertas y tomar las cápsulas por sus } \\
\text { extremos o nudos. }\end{array}$ \\
\hline 6 & Durante & $\begin{array}{l}\text { Conserve los elementos en la forma en que son } \\
\text { hallados, no altere el embalaje de los mismos, no } \\
\text { los abra. }\end{array}$ \\
\hline 7 & Durante & $\begin{array}{l}\text { Enumere los elementos en el mismo orden en } \\
\text { que son recuperados, colocándolos en una ban- } \\
\text { deja en donde sean visibles y le sean tomadas } \\
\text { fotografías. Se pueden limpiar con una compresa } \\
\text { húmeda. }\end{array}$ \\
\hline 8 & Durante & $\begin{array}{l}\text { Utilizar jeringas para recoger residuos líquidos } \\
\text { en aquellos casos en que se produzca el rompi- } \\
\text { miento accidental de la cápsula que contiene el } \\
\text { posible estupefaciente y depositar luego su con- } \\
\text { tenido en un frasco de vidrio. }\end{array}$ \\
\hline 9 & Durante & $\begin{array}{l}\text { Verificar el recipiente que contendrá los estupe- } \\
\text { facientes antes de ser embalado, debe estar com- } \\
\text { pletamente limpio y no debió ser usado antes. }\end{array}$ \\
\hline 10 & Después & $\begin{array}{l}\text { El hallazgo y la recuperación deben ser descritos } \\
\text { en forma detallada por el médico en nota adicio- } \\
\text { nal para la fiscalía. }\end{array}$ \\
\hline II & Después & $\begin{array}{l}\text { Los elementos extraídos deben entregarse al } \\
\text { personal de policía judicial una vez terminado el } \\
\text { acto quirúrgico. }\end{array}$ \\
\hline 12 & Después & $\begin{array}{l}\text { De no contarse con la presencia de un funciona- } \\
\text { rio de policía judicial los elementos se deben em- } \\
\text { balar en forma independiente en papel blanco y } \\
\text { limpio, rotulándolo y enumerando cada uno para } \\
\text { en bolsa de papel rotulada. }\end{array}$ \\
\hline 13 & Después & $\begin{array}{l}\text { Entregar a las autoridades de policía judicial con- } \\
\text { tada y pesada la sustancia con su respectiva cade- } \\
\text { na de custodia; copia de las fotografías o videos } \\
\text { realizados, pues además de asegurar el procedi- } \\
\text { miento y diligencia del operador de la salud, con- } \\
\text { tribuyen a verificar su fijación y localización y a } \\
\text { garantizar la autenticidad de la evidencia física. La } \\
\text { autoridad judicial puede posteriormente solicitar } \\
\text { copia de la historia clínica y de las radiografías en } \\
\text { cuyo caso deben estar disponibles para su entre- } \\
\text { ga oportuna. }\end{array}$ \\
\hline
\end{tabular}




\begin{tabular}{|c|c|l|}
\multicolumn{3}{|c}{ Tabla 5. Recomendaciones para material biológico } \\
\hline$N^{\circ}$ & $\begin{array}{c}\text { Momentos } \\
\text { quirúrgicos }\end{array}$ & \multicolumn{1}{c|}{ Recomendaciones } \\
\hline 1 & Antes & Examine el área que pueda poseer evidencias biológicas antes de la limpieza quirúrgica. \\
\hline 2 & Antes & $\begin{array}{l}\text { Si en el área se encuentran fluidos húmedos, recupérelos utilizando un hisopo de algodón o } \\
\text { gasa estéril, deje secar al medio ambiente y embálelo en tubo de ensayo (el hisopo) o en papel } \\
\text { limpio y estéril. }\end{array}$ \\
\hline 3 & Antes & Las muestras líquidas embálelas en tubos de ensayo bien tapados. \\
\hline
\end{tabular}

\section{Discusión}

Las recomendaciones establecidas por los expertos constituyen un documento guía y una propuesta de recuperación, manipulación, embalaje y entrega de la evidencia recuperada en cirugía y que representa un elemento probatorio en una investigación judicial.

Aquellos para estupefacientes superan en número a los demás tipos de evidencia, debido a que en la normatividad no hay especificaciones para el manejo de estos en las instituciones de salud y a la necesidad de responder ante la atención de pacientes identificados por la autoridades con este tipo de sustancias en su interior, en el tracto gastrointestinal o al interior de prótesis mamarias o glúteas. En el caso de las muestras biológicas por lo general se recuperan en pacientes con signos de acceso carnal violento.

Los procedimientos descritos responden a la necesidad de información específica sobre manejo de evidencia en las instituciones de salud, debido a la poca información que ofrece el manual de cadena de custodia. Representan una herramienta para la construcción de sus protocolos o guías en materia de pruebas obtenidas como producto de la atención de los pacientes quirúrgicos.

Los resultados del estudio responden a una necesidad imperante de los profesionales de la salud en especial aquellos que se desempeñan en el área quirúrgica y así ofrecer recursos que orienten su manera de proceder ante la identificación de una evidencia física o elemento materia de prueba en el cuerpo de un paciente, generando beneficios para los que han sido víctimas de hechos delictivos, dando cumplimiento a la normatividad respectiva y de esta manera evitar una implicación legal por manejo inadecuado.
Se reconoce como limitante del estudio la poca disponibilidad de personal experto en el área para participar en el proyecto y la pérdida de dos de ellos durante el estudio debido a que no respondieron los cuestionarios, a pesar de que fueron contactados en varias oportunidades.

\section{Conclusiones}

A partir de un consenso de expertos se logra establecer una propuesta de recomendaciones para la extracción, manipulación, embalaje y entrega de evidencias médicolegales extraídas de pacientes quirúrgicos en las instituciones de salud, de tal manera que se cumplan los requerimientos de la normatividad colombiana para mantener la cadena de custodia y evitar que los factores que intervienen en los procedimientos quirúrgicos afecten la autenticidad de los elementos materia de prueba.

\section{Referencias}

1. Dueñas Mendoza, L.Descripción epidemiológica del fenómeno de violencia interpersonal. Forensis. 2011: 13; 103-39.

2. Colombia. Congreso. Ley 906 de 2004 "Por la cual se expide el Código de Procedimiento Penal. (Corregida de conformidad con el Decreto 2770 de 2004)". Publicado en el Diario Oficial No. 45.658 de Septiembre 1 de 2004.

3. Colombia. Superintendencia Nacional de Salud. Circular externa 049 de abril 2 de 2008. Modificación a las instrucciones generales y remisión de información para la inspección, vigilancia y control contenidas en la Circular Externa número 047 (Circular única). Publicada en el Diario Oficial 46.951 de abril 05 de 2008.

4. Colombia. Fiscalía General. Resolución 2869 de 2003 (diciembre 29) por medio de la cual se adopta el Manual de Procedimientos del Sistema de Cadena de Custodia. Publicada en el Diario Oficial No. 45.424, de 8 de enero de 2004.

5. Colombia. Fiscalía General. Resolución 6394 de 2004 (diciembre 22) por medio de la cual se adopta el Manual de Procedimientos del Sistema de Cadena de Custodia para el sistema penal acusatorio. Publicada en el Diario Oficial 45772 de diciembre 24 de 2004.

6. Evans M, Stagner P. Maintaining the chain of custody: evidence handling in forensic cases. AORN J. 2003 Oct;78(4):563-9.

7. Olmos M, Sánchez R, Venegas M. Los consensos de expertos: una metodología útil en la toma de decisiones en salud. Rev Colomb Cancerol. 2006;10:50-60. 